\title{
Complicaciones de la histerectomía total laparoscópica en pacientes de un hospital de alta especialidad
}

\section{Complications of laparoscopic total hysterectomy in patients of a high specialty hospital}

\author{
Zarela L. Chinolla-Arellano', Jessica L. Bañuelos-Rodríguez², Viridiana Martínez-Sevilla² y \\ Juan A. García-Bello3* \\ ${ }^{1}$ Servicio de Medicina Materno Fetal; ${ }^{2}$ Servicio de Ginecología; ${ }^{3}$ División de Investigación. Unidad Médica de Alta Especialidad Hospital de Gineco- \\ Obstetricia No. 3 Dr. Víctor Manuel Espinoza de los Reyes Sánchez, Centro Médico Nacional La Raza, Instituto Mexicano del Seguro Social, \\ Ciudad de México, México
}

\begin{abstract}
Resumen
Objetivo: Describir las complicaciones transoperatorias y posoperatorias en pacientes operadas de histerectomía total laparoscópica. Método: Serie de casos del servicio de ginecología de la Unidad Médica de Alta Especialidad Hospital de GinecoObstetricia No. 3 del Centro Médico Nacional La Raza, del Instituto Mexicano del Seguro Social, de las pacientes sometidas a histerectomía total laparoscópica de diciembre de 2015 a diciembre de 2018. Se documentaron el peso uterino, el índice de masa corporal, la edad, la comorbilidad, el uso de dióxido de carbono y las complicaciones. Se usaron frecuencias, proporciones, medidas de tendencia central y dispersión. Resultados: Se reportan 79 pacientes de $44.2 \pm 7.5$ años, tiempo de cirugía de $104.3 \pm 32.7$ minutos y promedio de sangrado $102.8 \pm 62.3 \mathrm{ml}$. Presentaron complicaciones transoperatorias seis (7.6\%): dos (2.5\%) lesión vesical, una (1.3\%) lesión intestinal, una (1.3\%) hemorragia de muñón y dos (2.5\%) más conversión a laparotomía exploradora (una por tamaño uterino $>14 \mathrm{~cm}$ y otra por hallazgo de tumor ovárico de aspecto maligno). Siete pacientes (8.8\%) tuvieron complicaciones posoperatorias: cuatro (5.1\%) fueron reintervenidas (tres por hemorragia y una por dehiscencia de cúpula vaginal) y tres (3.7\%) tuvieron colección vaginal. Conclusiones: La tasa de complicaciones perioperatorias en esta serie coincide con la reportada en otros estudios. El tiempo quirúrgico y el sangrado fueron ligeramente menores, pese al poco tiempo de madurez del programa.
\end{abstract}

Palabras clave: Histerectomía. Laparoscopia. Complicaciones.

\section{Abstract}

Objective: Describe the intraoperative and postoperative complications in patients undergoing total laparoscopic hysterectomy (TLH). Method: Cases series carried out in the gynecology service of Hospital de Gineco-Obstetricia No. 3, Centro Medico Nacional La Raza, Instituto Mexicano del Seguro Social, of all patients undergoing total laparoscopic hysterectomy from December 2015 to December 2018. Uterine weight, body mass index, age, comorbidities, $\mathrm{CO}_{2}$ use, and complications were documented. Frequencies, proportions, central tendency and dispersion measures were used. Results: 79 patients are reported. Mean age was $44.2 \pm 7.5$ years. Surgery time was $104.3 \pm 32.7$ minutes, and bleeding $102.8 \pm 62.3 \mathrm{~mL}$. Six (7.6\%) had intraoperative complications: two (2.5\%) bladder injury, one (1.3\%) bowel injury, one (1.3\%) stump hemorrhage, and two (2.6\%) where converted to exploratory laparotomy: one for uterine size $>14 \mathrm{~cm}$, and one because malignant ovarian tumor

\section{Correspondencia:}

*Juan A. García-Bello

Calzada Vallejo, esquina Antonio Valeriano

Col. La Raza, Azcapotzalco

Fecha de recepción: 21-04-2020

C.P. 02990, Ciudad de México, México

E-mail: jagbello67@gmail.com
Cir Cir. 2021;89(3):347-353

Contents available at PubMed www.cirugiaycirujanos.com 0009-7411/@ 2020 Academia Mexicana de Cirugía. Publicado por Permanyer. Este es un artículo open access bajo la licencia CC BY-NC-ND (http://creativecommons.org/licenses/by-nc-nd/4.0/). 
was suspected. Seven patients (8.8\%) had postoperative complications: four (5.1\%) were re operated: 3 due to hemorrhage and 1 due to vaginal dome dehiscence; three (3.7\%) had vaginal collection. Conclusions: The rate of perioperative complications reported in this series coincides with that reported in other studies. Surgery time and bleeding were slightly minors regardless of program's youth.

Key words: Hysterectomy. Laparoscopy. Complications.

\section{Introducción}

La histerectomía es la cirugía ginecológica realizada con mayor frecuencia en todo el mundo, con millones de procedimientos realizados cada año'. En nuestro país no es posible estimar con precisión el número total y menos aún la vía de abordaje preferi$\mathrm{da}^{2}$. El primero en relacionar laparoscopia e histerectomía fue Semm, en 1984, pero la primera histerectomía total laparoscópica (HTL) fue publicada en 1989 por Harry Reich ${ }^{3}$.

Este procedimiento está indicado para enfermedad tanto benigna como maligna ${ }^{4}$. En un estudio realizado en el Instituto Nacional de Perinatología en 2009 se revisaron 555 histerectomías, de las cuales 226 fueron por vía laparoscópica, siendo la indicación más frecuente la miomatosis uterina $(58.6 \%)$, seguida de la hemorragia uterina anormal $(23.2 \%)$ y la adenomiosis $(18.2 \%)^{5}$, coincidiendo estos datos con los reportados en un estudio en España, en el que otras indicaciones reportadas con menor frecuencia fueron endometriosis, hemorragia uterina anormal, neoplasia intraepitelial cervical III persistente e hiperplasia con atipias $^{6}$.

Para el consentimiento de la HTL se deberá ofrecer información completa a las pacientes, haciendo particular énfasis en los beneficios, así como en los factores que podrían incrementar el riesgo de complicaciones durante y después del procedimiento quirúrgico7. La valoración del riesgo preoperatorio sirve como base para el asesoramiento apropiado de la paciente y los resultados posoperatorios ${ }^{8}$. Es importante considerar varios factores que pueden incidir en el resultado transoperatorio y posoperatorio, tales como el tamaño y el peso uterino ${ }^{9,10}$, el índice de masa corporal ${ }^{11}$, la edad y la presencia de comorbilidad ${ }^{12}$. Las contraindicaciones para la HTL hasta el momento son motivo de discusión. Las patologías con datos altamente sugestivos de proceso maligno, con sospecha de etapa clínica avanzada, hacen que una paciente no sea candidata a HTL. Fuera de estas circunstancias, no existe una contraindicación absoluta. Hoy en día, el principal factor limitante de la HTL es la inexperiencia de los cirujanos o el no contar con el recurso tecnológico adecuado para llevarla a cabo ${ }^{13}$.

Las principales ventajas de la HTL son un abordaje con incisiones menores, menor dolor posoperatorio, menos pérdida de sangre intraoperatoria que con el abordaje por vía laparotomía, y por lo tanto menor caída de la hemoglobina, reducción de la estancia hospitalaria, menor riesgo de infecciones de las heridas o de la pared abdominal, menor agresión inmunitaria, menor incidencia de hernias y, en consecuencia, una reincorporación más rápida a las actividades diarias ${ }^{10,14}$.

La principal desventaja es la necesidad de un mayor tiempo operatorio. Para hacer comparables los tiempos de la HTL y de la histerectomía abdominal, el tiempo quirúrgico promedio debería estar por debajo de 100 minutos. Esto se logra a medida que aumentan los casos y se incrementa la experiencia del equipo quirúrgico $0^{15}$. Las complicaciones, de acuerdo con el tiempo quirúrgico en que se producen, se pueden clasificar en complicaciones transoperatorias y posoperatorias.

Las complicaciones transoperatorias se pueden dividir, según el momento en que se presentan respecto al evento quirúrgico, en aquellas que se producen desde la introducción de los trocares y aquellas que ocurren propiamente durante el resto del procedimiento. Las primeras incluyen la insuflación extraperitoneal de dióxido de carbono, mientras que las segundas están relacionadas con lesiones vasculares, intestinales, vesicales, recto-vaginales o ureterales ${ }^{16,17}$, y con la conversión a laparotomía habitualmente secundaria a hemorragia masiva, dificultad de manipulación de tejidos, así como por problemas técnicos con el instrumental o el equipo biomédico utilizado, entre otros ${ }^{16-18}$.

Las complicaciones posoperatorias pueden ser generales (todas aquellas que pueden presentarse en cualquier proceder quirúrgico) o específicas de la HTL, como la colección intraabdominal ocasionada principalmente por sangrado, la formación de un hematoma en el área quirúrgica, la dehiscencia de alguno de los tejidos abordados, la formación de un 
absceso pélvico, el prolapso de la cúpula vaginal y la formación de fístulas ${ }^{19}$.

La HTL es una de las cirugías que han mostrado excelentes beneficios frente a la opción abierta ${ }^{20,21}$. Se trata de una técnica de reciente introducción en nuestro hospital (año 2015), por lo que nos era necesario evaluar los beneficios y las principales complicaciones de nuestras pacientes, así como tratar de identificar los principales factores de riesgo asociados para poder identificar áreas de oportunidad para mejorar incluso la preparación preoperatoria de las pacientes y con ello disminuir las complicaciones.

El objetivo de este estudio fue describir las complicaciones transoperatorias y posoperatorias en pacientes operadas de HTL en un hospital de alta especialidad.

\section{Método}

Previa obtención del número de registro institucional por el Comité Local de Investigación se realizó un estudio observacional, descriptivo y longitudinal en el servicio de ginecología de la Unidad Médica de Alta Especialidad Hospital de Gineco-Obstetricia No. 3 Dr. Víctor Manuel Espinosa de Los Reyes Sánchez, del Centro Médico Nacional La Raza, de pacientes que fueron operadas de HTL entre diciembre de 2015 y diciembre del 2018. De los registros clínicos se obtuvo información sobre indicación preoperatoria, edad, índice de masa corporal, peso uterino, comorbilidad, número y tipo de cirugías abdominales previas, insuflación extraperitoneal de dióxido de carbono, lesión a vasos sanguíneos, intestino, vejiga, uréteres o recto-vaginal, colección abdominal o de cúpula vaginal, prolapso de la cúpula vaginal, dehiscencia de la cúpula vaginal, fístulas, infección de herida quirúrgica, conversión a laparotomía exploradora (LAPE), días de estancia hospitalaria, sangrado, tiempo quirúrgico y cantidad de gas utilizado. Se describen frecuencias y proporciones, medias con desviaciones estándar 0 medianas con rangos e intervalos intercuartílicos.

Esta investigación es considerada como sin riesgo. Los procedimientos se apegan a las normas éticas internacionales, al Reglamento de la Ley General de Salud en materia de investigación para la salud y a la declaración de Helsinki y sus enmiendas. El balance riesgo-beneficio fue adecuado. En todo momento se preservará la confidencialidad de la información de las participantes; ni las bases de datos ni las hojas de colección contienen información que pudiera ayudar a identificarlas, y dicha información será conservada en registro aparte por el investigador principal bajo llave.

El $90 \%$ de las cirugías fueron realizadas por el mismo equipo quirúrgico. La técnica quirúrgica utilizada para la HTL en nuestras pacientes fue la siguiente: paciente en posición ginecológica, bajo anestesia general, con antisepsia de la región abdominal y genital, colocación de sonda de Foley y movilizador uterino tipo Apolo (HGO4), colocación de campos estériles y realización de incisión transumbilical, colocación de un trocar de $12 \mathrm{~mm}$ por técnica directa, neumoperitoneo con dióxido de carbono a $12 \mathrm{mmHg}$, visualización del sitio de entrada, visualización de la cavidad abdominal de $360^{\circ}$, sin datos de lesiones ni alteraciones abdominales, posición de Trendelemburg, introducción de trocares auxiliares, dos de lado izquierdo, en fosa iliaca izquierda, de $12 \mathrm{~mm}$, y otro de $5 \mathrm{~mm}$ paraumbilical homolateral (todos bajo visualización directa), introducción de trocar de $5 \mathrm{~mm}$ en la fosa ilíaca derecha, fulguración de ligamentos uterosacros con pinza selladora de vasos Ligasure ${ }^{\circledR}$, realización de colpotomía posterior con gancho monopolar, localización, fulguración y corte de salpinge izquierda con Ligasure ${ }^{\circledR}$, localización, fulguración y corte de ligamento útero-ovárico, localización, fulguración y corte de ligamento redondo, disección y corte de pliegue vésico-uterino con Ligasure ${ }^{\circledR}$ y gancho monopolar, descenso de vejiga, disección de hoja posterior de ligamento ancho, localización, fulguración y corte de arteria uterina con pinza Ligasure ${ }^{\circledR}$, se completó colpotomía lateral con gancho monopolar, misma técnica contralateral, extracción de pieza quirúrgica por vía vaginal, realización de puntos de Aldridge laparoscópicos extracorpóreos con sutura sintética absorbible (ácido poliglicólico $\mathrm{Vycril}^{\circledR} 1$ ), donde habitualmente fueron dos puntos de sutura suficientes para el cierre de la cúpula vaginal y en caso de que fuera necesario se dio un tercero, verificación de la hemostasia y de la integridad de la cúpula vaginal mediante un tacto gentil del segundo ayudante. Se procedió a realizar un lavado de tejidos con solución fisiológica y aspiración para verificar la hemostasia y retiro de contenido hemático. Se realizaron la extracción de los trocares bajo visión directa, la extracción del neumoperitoneo y el cierre de la pared abdominal de manera habitual. Aseo de la región y se concluyó el procedimiento.

\section{Resultados}

Se estudiaron los registros de 79 pacientes, que tuvieron una edad de $44.2 \pm 7.5$ años. El índice de masa 
Tabla 1. Pacientes con complicaciones transoperatorias

\begin{tabular}{|c|c|c|c|c|c|c|c|}
\hline Paciente & IMC & $\begin{array}{c}\text { Peso } \\
\text { uterino }(g)\end{array}$ & $\begin{array}{l}\text { Edad } \\
\text { (años) }\end{array}$ & Morbilidad & $\begin{array}{c}\text { Cirugías } \\
\text { previas } \\
\text { abdominales }\end{array}$ & Tipo de cirugías previas & Otro factor \\
\hline 1 Conversión a LAPE* & 28.3 & 240 & 43 & Ninguna & 0 & - & Ninguno \\
\hline 2 Conversión a $\mathrm{LAPE}^{\dagger}$ & 22.4 & 100 & 37 & Cáncer de mama & 0 & - & Ninguno \\
\hline $\begin{array}{l}3 \text { Conversión a LAPE y } \\
\text { lesión intestinal }{ }^{\ddagger}\end{array}$ & 31.2 & 100 & 49 & $\mathrm{DM} 2, \mathrm{HAC}, \mathrm{IRC}$ & 1 & OTB & $\begin{array}{l}\text { Miomatosis } \\
\text { uterina }\end{array}$ \\
\hline 4 Conversión a LAPE\$ & 51.8 & 160 & 45 & Ninguna & 1 & Apendicectomía & Ninguno \\
\hline $\begin{array}{l}5 \text { Conversión a LAPE y } \\
\text { lesión vesical }\end{array}$ & 25.7 & 150 & 43 & Cáncer de mama & 3 & Cesárea (2) y LAPE (1) & Ninguna \\
\hline 6 Lesión vesical & 22.1 & 220 & 47 & $\begin{array}{l}\text { Uropatía } \\
\text { obstructiva }\end{array}$ & 4 & $\begin{array}{l}\text { Cesárea (1) y } \\
\text { nefrostomías (3) }\end{array}$ & $\begin{array}{l}\text { Miomatosis } \\
\text { uterina }\end{array}$ \\
\hline
\end{tabular}

DM2: diabetes mellitus tipo 2; HAC: hipertensión arterial crónica; IMC: índice de masa corporal; IRC: insuficiencia renal crónica; LAPE: laparotomía exploradora; OTB: oclusión tubárica bilateral.

*Por hallazgo incidental de tumor de ovario bilateral de aspecto maligno.

†Por hemorragia.

†Para reparación de serosa intestinal.

\$Por gran tamaño uterino.

corporal promedio fue de $25.7 \pm 3.5$. La principal indicación de la HTL fue miomatosis uterina en 28 casos (35.9\%), en segundo lugar el sangrado uterino anormal con 17 casos (21.8\%), y en tercero, con 11 casos, la hiperplasia endometrial (14.1\%). Dos pacientes tuvieron como indicación de la HTL un cáncer cérvico-uterino y una paciente tuvo sangrado uterino anormal. El tiempo de cirugía fue de $104.3 \pm 32.7$ minutos, la cantidad promedio de dióxido de carbono por cirugía para el neumoperitoneo fue de $127.5 \pm 63.5$ I y la cantidad promedio de sangrado fue de $102.8 \pm 62.3 \mathrm{ml}$. En cuanto al tamaño uterino promedio, fue de $9.76 \pm 2.0 \mathrm{~cm}$. El tiempo promedio de estancia hospitalaria fue de $3.1 \pm 1.3$ días. Solo hubo cinco casos de conversión a laparotomía por lesión de órgano vecino (intestinal y vesical).

Se presentaron seis complicaciones transoperatorias, y la principal fue la conversión a LAPE, que fue necesaria en cinco casos (6.3\%). De estas, una fue secundaria a lesión vesical, otra por lesión de colon (ambas con conversión a LAPE para reparación), una por hemorragia de pedículo (ya que la pinza selladora de vaso no logró mitigar el sagrado), una por tamaño uterino $>14 \mathrm{~cm}$ y una por hallazgo quirúrgico de un tumor de ovario con aspecto maligno. Además, hubo una lesión vesical reparada durante el mismo procedimiento laparoscópico (Tabla 1). De las seis pacientes que presentaron complicaciones transoperatorias, solo una padecía hipertensión arterial crónica, diabetes mellitus tipo 2 e insuficiencia renal crónica; el resto de las pacientes no tenían comorbilidad.
Siete pacientes (8.8\%) tuvieron alguna complicación posoperatoria, de las cuales cuatro requirieron reintervensión quirúrgica: tres por hemorragia y una por dehiscencia de cúpula vaginal. En las otras tres pacientes la complicación fue colección vaginal (Tabla 2).

Durante el seguimiento, una vez egresadas las pacientes, se detectaron en tres de ellas colecciones en la cúpula vaginal, las cuales fueron drenadas por vía vaginal. De las cuatro pacientes que requirieron reintervención quirúrgica, en tres la indicación fue miomatosis y en una fue sangrado uterino anormal.

\section{Discusión}

En un estudio realizado por Morgan, et al. ${ }^{15}$ en 87 pacientes operadas de 2003 a 2006, la indicación más frecuente de histerectomía fue sangrado uterino anormal, en el $79.3 \%$, seguido de miomatosis uterina en el $63.7 \%$, hiperplasia endometrial en el $10.1 \%$, dolor pélvico crónico en el $10.3 \%$, tumor anexial en el $6.8 \%$ y lesión escamosa intraepitelial del cuello uterino de alto grado en el $3.4 \%$, lo que difiere poco con lo observado en nuestro estudio, en el que la principal indicación de HTL fue la presencia de miomatosis uterina seguida del sangrado uterino anormal. Así mismo, nuestros resultados son similares a los de Maryland en 5660 pacientes, donde el $65.9 \%$ de las pacientes tenían la indicación de histerectomía por fibromas y el $57.5 \%$ por sangrado uterino ${ }^{12}$. 
Tabla 2. Pacientes con complicaciones posoperatorias

\begin{tabular}{|c|c|c|c|c|c|c|c|}
\hline $\begin{array}{l}\text { Paciente y } \\
\text { complicación }\end{array}$ & IMC & $\begin{array}{l}\text { Peso } \\
\text { uterino }(\mathrm{g})\end{array}$ & $\begin{array}{l}\text { Edad } \\
\text { (años) }\end{array}$ & Morbilidad & $\begin{array}{l}\text { Cirugías previas } \\
\text { abdominales }\end{array}$ & Tipo de cirugías previas & Otro factor \\
\hline $\begin{array}{l}1 \text { Formación de } \\
\text { hematoma }\end{array}$ & 34.1 & 90 & 46 & Ninguna & 0 & Ninguna & $\begin{array}{l}\text { Cáncer } \\
\text { cervicouterino }\end{array}$ \\
\hline $\begin{array}{l}2 \text { Formación de } \\
\text { hematoma }\end{array}$ & 24.6 & 100 & 34 & Ninguna & 2 & Cesáreas & $\begin{array}{l}\text { Cáncer } \\
\text { cervicouterino }\end{array}$ \\
\hline $\begin{array}{l}3 \text { Absceso } \\
\text { cúpula vaginal }\end{array}$ & 24.8 & 120 & 54 & Ninguna & 2 & OTB, colecistectomía & Miomatosis uterina \\
\hline $\begin{array}{c}4 \text { Reintervención } \\
\text { quirúrgica* }\end{array}$ & 24.0 & 180 & 49 & Ninguna & 0 & Ninguna & Miomatosis uterina \\
\hline $\begin{array}{c}5 \text { Reintervención } \\
\text { quirúrgica }^{\dagger}\end{array}$ & 22.1 & 220 & 47 & Ninguna & 4 & $\begin{array}{l}\text { Cesárea (1) y } \\
\text { nefrostomías (3) }\end{array}$ & Miomatosis uterina \\
\hline $\begin{array}{l}6 \text { Reintervención } \\
\text { quirúrgica }\end{array}$ & 21.8 & 80 & 31 & SAAF & 1 & Cesárea & Miomatosis uterina \\
\hline $\begin{array}{c}7 \text { Reintervención } \\
\text { quirúrgica§ }\end{array}$ & 31.2 & 100 & 49 & $\begin{array}{l}\text { DM2, HAC, } \\
\text { IRC }\end{array}$ & 1 & OTB & Miomatosis uterina \\
\hline \multicolumn{8}{|c|}{$\begin{array}{l}\text { DM2: diabetes mellitus tipo 2; HAC: hipertensión arterial crónica; IMC: índice de masa corporal; IRC: insuficiencia renal crónica; OTB: oclusión tubárica bilateral; SAAF: síndrome de } \\
\text { anticuerpos antifosfolípidos. } \\
\text { *Por hemorragia de cúpula vaginal y ligamento infundibulopélvico derecho. } \\
\text { †Por hematoma disecante. } \\
\text { fPor dehiscencia total de cúpula vaginal. }\end{array}$} \\
\hline
\end{tabular}

El tiempo operatorio depende mucho de la experiencia y de la cantidad de cirugías laparoscópicas realizadas por año, que son clasificadas según la Sociedad Internacional de Endoscopia Ginecológica ${ }^{8}$ en menos de 100 , entre 100 y 200 , y más de 200 . En nuestro grupo, con apenas 79 cirugías realizadas, logramos un tiempo quirúrgico promedio de 104.3 minutos, algo menor que en los estudios de Morgan, et al. $^{15}$, en el que se reporta una media de $123.0 \mathrm{mi}-$ nutos, y de Ayala, et al. ${ }^{5}$, con 139.3 minutos.

En nuestro estudio, la pérdida sanguínea fue menor que la reportada en otros estudios, con una media de $102.8 \mathrm{ml}$, frente a una media de $151.0 \mathrm{ml}$ reportada por Morgan, et al. ${ }^{15} \mathrm{y}$ de $119.7 \mathrm{ml}$ por Ayala, et al. ${ }^{5}$, pero el tamaño uterino en nuestro estudio fue de $9.76 \mathrm{~cm}$ en promedio, menor que el reportado en otros estudios de $14.54 \mathrm{~cm}^{15}$ y $12.4 \mathrm{~cm}^{22}$. Por otro lado, el promedio de estancia hospitalaria fue de 3.1 días, similar a lo reportado en otros estudios ${ }^{15,23}$. Una revisión Cochrane que examinó 47 ensayos controlados aleatorizados demostró que la histerectomía mínimamente invasiva realizada para indicaciones benignas tuvo como resultado un retorno más rápido a las actividades normales para las pacientes en comparación con la histerectomía abdominal. ${ }^{24}$

La frecuencia de complicaciones perioperatorias encontradas en nuestro estudio fue del $16.4 \%$, de las cuales el $7.5 \%$ correspondieron a complicaciones intraoperatorias y el $8.8 \%$ a complicaciones posoperatorias. Los resultados son muy similares a los reportados por De los Ríos, et al..$^{14}$ en 2009, en cuyo estudio la frecuencia global de complicaciones fue del $12.5 \%$, de las cuales el $11.4 \%$ correspondieron a complicaciones transoperatorias y el $1.1 \%$ a posoperatorias; y un poco mayores que los reportados por Argüello, et al..$^{25}$ en 2012, quienes hallaron una frecuencia global de complicaciones del $5.6 \%$, de las cuales el $0.5 \%$ correspondieron a complicaciones intraoperatorias y el $5.1 \%$ a complicaciones posoperatorias.

Las principales complicaciones transoperatorias en nuestro estudio fueron la lesión vesical y la conversión a laparotomía, con una frecuencia de presentación del $2.5 \%$ y el $6.3 \%$, respectivamente. En ambos casos, la indicación de la HTL fue miomatosis uterina (en la cara anterior del útero) y existía el antecedente de cesáreas. Corvalán, et al. ${ }^{26}$, en 2004, reportaron una tasa de conversión a laparotomía similar a la nuestra. En nuestro estudio, las causas de conversión a laparotomía fueron la hemorragia y la lesión de órgano vecino (vejiga e intestino).

En el mismo estudio de Corvalán et al. $^{26}$, de 139 pacientes operadas de HTL, el $48.9 \%$ presentaban cirugías previas, de las cuales el $15.3 \%$ correspondían a cesáreas, el 15.1\% a cirugías ginecológicas 
y el $18.5 \%$ a cirugías no ginecológicas, lo que es relevante para los resultados de la HTL debido a la posibilidad de encontrar adherencias que dificultan la técnica. Sin embargo, las complicaciones asociadas a este factor durante el procedimiento laparoscópico no fueron relevantes en esa serie, excepto por las pacientes que presentaron lesión vesical que tenían el antecedente de cesáreas. En nuestro estudio, el antecedente de cirugías previas, en su mayoría cesáreas, se presentó con frecuencia.

En otro estudio, Murillo, et al. ${ }^{22}$, en el Hospital Español de México, realizaron 3924 histerectomías en un periodo de 10 años. De ellas, el $83.1 \%(n=3260)$ se efectuaron por laparotomía, el $7.3 \%(n=288)$ por vía vaginal y el $9.5 \%(n=376)$ por vía laparoscópica. El grupo con mayor porcentaje de cesáreas previas fue el de histerectomía supracervical laparoscópica (55.5\%), en comparación con los grupos de histerectomía laparoscópica (49\%) e histerectomía vaginal asistida por laparoscopia (41.5\%), lo cual confirma que los procedimientos son posibles incluso en estas pacientes y, por tanto, el acceso laparoscópico también las beneficia.

En relación con las lesiones vesicales, se estima que se producen lesiones del tracto genitourinario con una frecuencia del 1-2\% de todas las cirugías ginecológicas mayores, y se calcula que el $75 \%$ de estas lesiones se producen durante la histerectomía ${ }^{26}$. Las cirugías abdominales previas y la presencia de miomas cervicales aumentaron el riesgo de lesiones en las vías urinarias ${ }^{22,28,29}$.

En nuestra serie se presentaron dos casos de lesión vesical y un caso de lesión intestinal, las cuales fueron reconocidas y reparadas sin complicaciones de manera transoperatoria y sin secuelas. Un estudio de CipuIlo, et al. ${ }^{29}$ realizado en 2008 destaca la gran variabilidad entre los diferentes centros hospitalarios, con una frecuencia de complicaciones urológicas de entre el 0,4\% y el $4 \%$. Las lesiones del techo de la vejiga no son exclusivas de la HTL y ocurren también frecuentemente en la histerectomía abdominal, aunque la frecuencia es mucho mayor en la HTL ( 2 vs. $0,8 \%$ ).

El citado estudio en Maryland reporta que la comorbilidad identificada está significativamente asociada con la mortalidad hospitalaria e incluye afecciones agudas y crónicas ${ }^{12}$. En nuestro estudio, de las seis pacientes que presentaron complicaciones transoperatorias, solo una padecía hipertensión arterial crónica, diabetes mellitus tipo 2 e insuficiencia renal crónica; el resto no tenían comorbilidad. Por lo anterior, no es posible concluir si la comorbilidad de las pacientes fue un factor de riesgo significativo para la presencia de complicaciones transoperatorias.

Las principales complicaciones posoperatorias que se presentaron en nuestro estudio fueron la formación de colección vaginal y la reintervención quirúrgica. Las estimaciones de la incidencia de abscesos después de la histerectomía van desde el $19.4 \%$ hasta el $90 \%{ }^{27}$. En nuestro estudio solo hubo tres casos de formación de colección vaginal: una de ellas fue un absceso en la cúpula vaginal y dos fueron casos de formación de hematoma en la cúpula vaginal, los cuales se resolvieron favorablemente. La segunda complicación posoperatoria más frecuente en este estudio fue la reintervención quirúrgica, realizada en cuatro pacientes, tres de ellas por abdomen agudo secundario a hemorragia y una por dehiscencia de cúpula vaginal, ameritando reforzamiento de pedículos y cierre de cúpula vaginal en un segundo tiempo quirúrgico mediante laparotomía; afortunadamente, las pacientes continuaron la recuperación posoperatoria sin más complicaciones. Cabe mencionar que la misma paciente con antecedente de diabetes mellitus tipo 2 , nefropatía e hipertensión arterial sistémica crónica presentó varias complicaciones (sangrado transquirúrgico, conversión a LAPE por lesión intestinal y reintervención por sangrado en el posoperatorio). En el estudio de Maryland se reporta una tasa de hemorragia posoperatoria del $0.8 \%$, menor que la observada en nuestro estudio ${ }^{12,14}$.

En nuestro estudio, solo dos de las siete pacientes con complicaciones posoperatorias tenían comorbilidad: una de ellas padecía síndrome de anticuerpos antifosfolípidos y la otra hipertensión arterial sistémica, diabetes mellitus tipo 2 e insuficiencia renal crónica.

El número de cirugías previas es un factor de riesgo constante para presentar complicaciones, tanto transoperatorias como posoperatorias. Observamos cinco casos de complicaciones posoperatorias en las pacientes con más de una cirugía previa, siendo las cesáreas y la miomatosis uterina los factores encontrados con mayor frecuencia en las pacientes que necesitaron reintervención.

Consideramos importante destacar que en nuestro estudio hubo una menor cantidad de sangrado y un menor tiempo quirúrgico que en el resto de los estudios revisados, lo cual puede explicarse por el hecho de que todas las cirugías fueron realizadas por el mismo equipo quirúrgico (cirujano y ayudante). Creemos que lo anterior nos permite hacer un análisis de la experiencia en esta técnica quirúrgica. 


\section{Conclusiones}

Pese a que, por definición, somos un centro hospitalario con menos de 100 cirugías laparoscópicas por año, la tasa de complicaciones perioperatorias en este estudio coincide con la reportada por otros autores.

Los factores más frecuentemente asociados a complicaciones transoperatorias, como lesión a órgano vecino (vejiga e intestino) y necesidad de conversión a laparotomía, fueron la presencia de cirugías abdominales previas y la miomatosis uterina.

La complicación posoperatoria más frecuente fue la colección en el fondo de saco, con tres casos; en dos de ellos la indicación de la HTL fue cáncer cérvico-uterino y en otro caso fue sangrado uterino anormal.

Así mismo, se observó un excelente resultado en comparación con estudios realizados en otras instituciones, con un menor tiempo quirúrgico y menos sangrado transoperatorio, así como una menor tasa de complicaciones perioperatorias. Esto puede deberse a que todas las cirugías reportadas fueron realizadas por el mismo equipo del servicio de ginecología del hospital.

\section{Responsabilidades éticas}

Protección de personas y animales.Los autores declaran que los procedimientos seguidos se conformaron a las normas éticas del comité de experimentación humana responsable y de acuerdo con la Asociación Médica Mundial y la Declaración de Helsinki.

Confidencialidad de los datos. Los autores declaran que han seguido los protocolos de su centro de trabajo sobre la publicación de datos de pacientes.

Derecho a la privacidad y consentimiento informado. Los autores declaran que en este artículo no aparecen datos de pacientes.

\section{Conflicto de intereses}

Los autores manifiestan que no tienen ningún conflicto de intereses respecto de este manuscrito.

\section{Bibliografía}

1. Indicaciones y contraindicaciones de la histerectomía en el segundo nivel de atención. México: Secretaría de Salud; 2010. Disponible en: http://www. cenetec.salud.gob.mx/descargas/gpc/CatalogoMaestro/454_GPC_Histerectomxa_segundo_nivel/SSA-295-10_Histerectomxa_-_RER_xCorregidax.pdf

2. Noguera M, Briones J, Rabadán C, Antonio P, Bautista E, Ceja J. La histerectomíaa laparoscópica como procedimiento de primera línea en el tratamiento de mujeres con patología benigna del útero. Ginecol Obstet Mex. 2013;81:448-53.
3. Seminario J. Histerectomía laparoscópica. Rev Per Ginecol Obstet. 2009;55:93-99.

4. Kauko M. New techniques using the ultrasonic scalpel in laparoscopic hysterectomy. Curr Opin Obstet Gynecol. 1998;10:303-5.

5. Ayala R, Briones C, Anaya H, Leory L, Zavaleta R. Histerectomía total laparoscópica: estudio descriptivo de la experiencia institucional con 198 casos. Ginecol Obstet Mex. 2010;78:605-11.

6. Payá V, Diago V, Abad A, Costa S, Coloma F, Martín-Vallejo J, et al. Histerectomía laparoscópica frente a histerectomía abdominal: estudio clínico comparativo. Clin Invest Gin Obstet. 2002;29:284-9.

7. NICE Clinical Guideline. Heavy menstrual bleeding. National Collaboration Centre for Women's and Children's Health, commissioned by the National Institute for Health and Clinical Excellence. Disponible en: https://www.rcog.org.uk/en/guidelines-research-services/guidelines/heavy-menstrual-bleeding-nice-clinical-guideline-44/

8. ACOG Practice Bulletin No. 65. Management of endometrial cancer. The American College of Obstetricians and Gynecologists. Disponible en: https://www.ncbi.nlm.nih.gov/pubmed/16055605

9. Sardiñas R, Fernández L. Twelve year experience in performing laparoscopic hysterectomy. Revista Cubana de Cirugía. 2014;53:281-8.

10. Sardiñas PR. La histerectomía laparoscópica y sus aspectos fundamentales. Revista Cubana de Cirugía. 2015;54:82-95.

11. Castañeda J, De los Ríos J, Calle G, Serna E, Vázquez R, Mejía A, et al. The association between body mass index and perioperative and postoperative outcomes in patients undergoing total laparoscopic hysterectomy. Rev Colomb Obstet Ginecol. 2010;61:108-12.

12. Metha A, Xu T, Hutfless S, Makary M, Sinno A, Tanner E, et al. Patient, surgeon and hospital disparities associated with benign hysterectomy approach and perioperative complications. Am J Obstet Gynecol. 2017;216:497.e1-10

13. Thoma V, Salvatores M, Mereu L, Chau I, Wattiez A. Histerectomía laparoscópica: técnica e indicaciones. EMC - Urología. 2007;39:1-9.

14. De los Ríos J, Castañeda J, Calle G, Serna E, Vázquez R, Aragón A, et al. Histerectomía laparoscópica total en la unidad de endoscopia ginecológica de la Clínica del Prado, Medellín (Colombia), 2002-2008. Rev Colomb Obstet Ginecol. 2009;60:320-7.

15. Morgan O, López M, Elorriaga E, Soto J, Lelevier H. Histerectomía total laparoscópica: complicaciones y evolución clínica en una serie de 87 casos. Ginecol Obstet Mex. 2008;76:520-5

16. Lobato MJL, Villasante MA, Pérez SC, Marqués MM, López VM. Evisceración intestinal transvaginal después de histerectomía. Revista Cubana de Cirugía. 2010;49:69-73.

17. Ricari $E$, Oroz L, Lara A. Complicaciones de la cirugía ginecológica. An Sist Sanit Navar. 2009;32:65-79.

18. Meraz D, Rodríguez S, Ramírez C, Escobar L, Quiroz G. Lesiones ureterales atendidas en el Instituo Nacional de Perinatología. Ginecol Obstet Mex. 2007;75:187-92.

19. Sardiñas P. Histerectomía laparoscópica: experiencia en el Hospital Clínico Quirúrgico Hermanos Ameijeiras. [Tesis]. La Habana: Universidad de Ciencias Médicas de La Habana; 2013.

20. Härkki-Siren P, Sjöberg J, Kurki T. Major complications of laparoscopy: a follow-up Finnish study. Obstet Gynecol. 1999;54:632-4.

21. Makinen J, Johansson J, Tomas C, Tomás E, Heinonen $P$, Laatikainen T, et al. Morbidity of 10110 hysterectomies by type of approach. Hum Reprod. 2001;16:1473-8.

22. Murillo J, Pedraza L, Aguirre X, López P. Histerectomía por laparoscopia: experiencia de 10 años en el Hospital Español de México. Ginecol Obstet Mex. 2007;75:667-77.

23. Zarhi J, Toso J, Cifuentes J, Vacca F, Stuardo P. Cirugía endoscópica ginecológica: experiencia en 8 años. Rev Chil Obstet Ginecol. 2004;69:279-85.

24. Aarts JWM, Nieboer TE, Johnson N, Tavender E, Garry R, Mol BWJ, et al. Surgical approach to hysterectomy for benign gynaecological disease. Cochrane Database of Syst Rev 2015 (8): CD003677. doi: 10.1002/14651858.CD003677.pub5.

25. Argüello-Argüello R. Complicaciones en 748 histerectomías por laparoscopia utilizando un manipulador uterino con resaltador vaginal. Rev Colomb Obstet Ginecol. 2012;63:252-8.

26. Corvalán J, Roos A, Lattus J, Barrera V, Gallardo E, Flores J, et al. Histerectomía total laparoscópica. Resultados en 3 años de desarrollo de la técnica utilizando el manipulador uterino de Clermont Ferrand. Rev Chil Obstet Ginecol. 2004;69:446-50.

27. Clarke-Pearson D, Geller E. Complicaciones de la histerectomía. Obstet Gynecol. 2013;121:654-73.

28. Garrett J, Nascimento C, Nicklin L, Perrin C, Obermair A. Total laparoscopic hysterectomy: the Brisbane learning curve. Aust N Z J Obstet Gynaecol. 2007;47:65-9.

29. Cipullo L, Cassese S, Fasolino L, Fasolino A. Laparoscopic hysterectomy and urological lesions: risk analysis based on current literature and preventive strategies. Minerva Ginecol. 2008;60:331-7. 